\title{
MRI characteristics of neuromyelitis optica spectrum disorder
}

\author{
An international update
}

Ho Jin Kim, MD, PhD

Friedemann Paul, MD

Marco A. Lana-Peixoto, $\mathrm{MD}, \mathrm{PhD}$

Silvia Tenembaum, MD

Nasrin Asgari, MD, PhD

Jacqueline Palace, DM, FRCP

Eric C. Klawiter, MD

Douglas K. Sato, MD

Jérôme de Seze, MD,

$\mathrm{PhD}$

Jens Wuerfel, MD

Brenda L. Banwell, MD

Pablo Villoslada, MD

Albert Saiz, MD

Kazuo Fujihara, MD, $\mathrm{PhD}$

Su-Hyun Kim, MD

With The Guthy-Jackson

Charitable Foundation

NMO International

Clinical Consortium \&

Biorepository

Correspondence to

Dr. Ho Jin Kim:

hojinkim@ncc.re.kr

Supplemental data at Neurology.org

\section{ABSTRACT}

Since its initial reports in the 19th century, neuromyelitis optica (NMO) had been thought to involve only the optic nerves and spinal cord. However, the discovery of highly specific antiaquaporin-4 antibody diagnostic biomarker for NMO enabled recognition of more diverse clinical spectrum of manifestations. Brain MRI abnormalities in patients seropositive for anti-aquaporin-4 antibody are common and some may be relatively unique by virtue of localization and configuration. Some seropositive patients present with brain involvement during their first attack and/or continue to relapse in the same location without optic nerve and spinal cord involvement. Thus, characteristics of brain abnormalities in such patients have become of increased interest. In this regard, MRI has an increasingly important role in the differential diagnosis of NMO and its spectrum disorder (NMOSD), particularly from multiple sclerosis. Differentiating these conditions is of prime importance because early initiation of effective immunosuppressive therapy is the key to preventing attack-related disability in NMOSD, whereas some disease-modifying drugs for multiple sclerosis may exacerbate the disease. Therefore, identifying the MRI features suggestive of NMOSD has diagnostic and prognostic implications. We herein review the brain, optic nerve, and spinal cord MRI findings of NMOSD. Neurology ${ }^{\circledR} 2015 ; 84: 1165-1173$

\section{GLOSSARY}

AQP4 = aquaporin-4; lgG = immunoglobulin G; LETM = longitudinally extensive transverse myelitis; $\mathbf{M O G}$ = myelin-oligodendrocyte glycoprotein; $\mathbf{M S}$ = multiple sclerosis; $\mathbf{N M O}$ = neuromyelitis optica; $\mathbf{N M O S D}=$ neuromyelitis optica spectrum disorder; $\mathbf{O N}=$ optic neuritis.

Neuromyelitis optica (NMO) is an inflammatory disease of the CNS that is characterized by severe attacks of optic neuritis $(\mathrm{ON})$ and longitudinally extensive transverse myelitis (LETM). ${ }^{1}$ The past decade has witnessed dramatic advances in our understanding of NMO. Such advances were initiated by the discovery of the disease-specific autoantibody, NMO-immunoglobulin G (NMO-IgG), and subsequent identification of the main target autoantigen, aquaporin- 4 (AQP4), which has distinguished $\mathrm{NMO}$ as a distinct disease from multiple sclerosis (MS). ${ }^{2}$

Current diagnostic criteria, however, still require both $\mathrm{ON}$ and myelitis for an NMO diagnosis. ${ }^{3}$ Nevertheless, the identification of anti-AQP4 antibodies beyond the current diagnostic criteria of $\mathrm{NMO}$ indicates a broader clinical phenotype of this disorder, so-called "NMO spectrum disorder" (NMOSD). ${ }^{4,5}$ The NMOSD encompasses anti-AQP4 antibody seropositive patients with limited or inaugural forms of NMO and with specific brain abnormalities. It also includes anti-AQP4 antibody seropositive patients with other autoimmune disorders such as systemic

From the Department of Neurology (H.J.K., S.-H.K.), Research Institute and Hospital of National Cancer Center, Goyang, Korea; NeuroCure Clinical Research Center and Clinical and Experimental Multiple Sclerosis Research Center (F.P., J.W.), Department of Neurology, Charité University Medicine, Berlin, Germany; CIEM MS Research Center (M.A.L.-P.), Federal University of Minas Gerais Medical School, Belo Horizonte, Brazil; Department of Neurology (S.T.), National Paediatric Hospital Dr. Juan P. Garrahan, Buenos Aires, Argentina; Neurobiology (N.A.), Institute of Molecular Medicine, University of Southern Denmark; Department of Neurology (N.A.), Vejle Hospital, Denmark; Department of Clinical Neurology (J.P.), John Radcliffe Hospital, Oxford, UK; Department of Neurology, Massachusetts General Hospital (E.C.K.), Harvard Medical School, Boston, MA; Department of Neurology (D.K.S.), Tohoku University School of Medicine, Sendai, Japan; Neurology Department (J.d.S.), Hôpitaux Universitaires de Strasbourg, France; Institute of Neuroradiology (J.W.), University Medicine Goettingen, Germany; Department of Pediatrics (B.L.B.), Division of Neurology, The Children's Hospital of Philadelphia; Department of Neurology (B.L.B.), The University of Pennsylvania; Center of Neuroimmunology (P.V., A.S.), Service of Neurology, Hospital Clinic and Institute of Biomedical Research August Pi Sunyer, Barcelona, Spain; and Department of Multiple Sclerosis Therapeutics (K.F.), Tohoku University Graduate School of Medicine, Sendai, Japan.

The Guthy-Jackson Charitable Foundation NMO International Clinical Consortium \& Biorepository coinvestigators are listed on the Neurology ${ }^{\circledR}$ Web site at Neurology.org.

Go to Neurology.org for full disclosures. Funding information and disclosures deemed relevant by the authors, if any, are provided at the end of the article. 
lupus erythematosus and Sjögren syndrome. ${ }^{4}$ In this regard, MRI has an increasingly important role in differentiating NMOSD from other inflammatory disorders of the CNS, particularly from MS.6,7 Differentiating these conditions is critical because treatments are distinct. Furthermore, recent advanced MRI techniques are detecting additional specific markers and help elucidate the underlying mechanisms of tissue damage in NMOSD.

We herein summarize the MRI findings of NMOSD and discuss their diagnostic and prognostic implications.

BRAIN MRI FINDINGS IN NMOSD Since the early studies using brain MRI in NMO, ${ }^{8,9}$ unexplained clinically silent and nonspecific white matter abnormalities were found in some patients. With the advent of AQP4-IgG assays, it became clear that a high proportion of patients with NMOSD harbored brain MRI abnormalities, frequently located in areas associated with high AQP4 expression. ${ }^{10,11}$ However, brain abnormalities also occurred in areas where AQP4 expression is not particularly high. ${ }^{12}$ Although nonspecific small dots and patches of hyperintensity in subcortical and deep white matter on T2-weighted or fluid-attenuated inversion recovery sequences are the most common findings in NMOSD, certain lesions have a location or appearance characteristic for NMOSD. ${ }^{6,711-15}$

Before the discovery of anti-AQP4 antibody, brain MRI abnormalities were reported in only $13 \%$ to $46 \%$ of patients with NMO.${ }^{1,8,16}$ However, when excluding the brain MRI criteria, the incidence of brain MRI abnormalities increased to $50 \%$ to $85 \%$ using the revised $2006 \mathrm{NMO}$ diagnostic criteria ${ }^{3,11,13,17, \mathrm{el}-\mathrm{e} 3}$ and to $51 \%$ to $89 \%$ in seropositive patients with NMOSD. ${ }^{5,12,18,19, e 4, e 5}$ Furthermore, brain MRI abnormalities at onset have been reported in $43 \%$ to $70 \%$ of patients with NMOSD. ${ }^{5,7,11}$ One of the explanations for discrepancies in frequency between studies may be that brain MRI abnormalities become more frequent with duration of disease. In a published series of 88 seropositive children, brain abnormalities were observed in $68 \%$ of the children with available MRI studies, and were predominantly located within periventricular regions of the third (diencephalic) and fourth ventricles (brainstem), supratentorial and infratentorial white matter, midbrain, and cerebellum. ${ }^{20}$ This is consistent with the observation that $45 \%$ to $55 \%$ of children with NMOSD show episodic cerebral symptoms, including ophthalmoparesis, intractable vomiting and hiccups, altered consciousness, severe behavioral changes, narcolepsy, ataxia, and seizures. ${ }^{20}$
Classification of brain MRI findings seen in NMOSD. Periependymal lesions surrounding the ventricular system. Diencephalic lesions surrounding the third ventricles and cerebral aqueduct. Diencephalic lesions surrounding the third ventricles and cerebral aqueduct, which include the thalamus, hypothalamus, and anterior border of the midbrain have been reported in NMOSD (figure 1A). ${ }^{10,12}$ These lesions frequently are asymptomatic, but some patients may present with a syndrome of inappropriate antidiuretic hormone secretion, ${ }^{\mathrm{e} 6}$ narcolepsy, ${ }^{\mathrm{e} 7}$ hypothermia, hypotension, hypersomnia, obesity, ${ }^{\mathrm{e} 8}$ hypothyroidism, hyperprolactinemia, secondary amenorrhea, galactorrhea, and behavioral changes. $^{\text {e9 }}$

Dorsal brainstem lesions adjacent to the fourth ventricle. One of the most specific brain MRI abnormalities in patients with NMOSD is a lesion in the dorsal brainstem adjacent to the fourth ventricle including the area postrema and the nucleus tracts solitarius. Such lesions are highly associated with intractable hiccups, nausea, and vomiting, ${ }^{10,12,21}$ and have been reported in $7 \%$ to $46 \%$ of patients with NMOSD. ${ }^{12,15, \mathrm{e} 1, \mathrm{e} 10}$ This area, the emetic reflex center, has a less restrictive blood-brain barrier, making it more accessible to AQP4-IgG attack. The MRI as well as clinical evidence support the notion that area postrema is an important point of attack in patients with NMOSD and further suggests that this area is a portal for entry of circulating IgG into the CNS. ${ }^{22,23}$ Pathologic abnormalities were noted in this region in $40 \%$ of patients with NMO, but there was no obvious neuronal, axonal, or myelin loss. ${ }^{21}$ Medullary lesions are often contiguous with cervical cord lesion, usually taking a linear shape (figure 1B.b). These lesions may be associated with the first symptoms of the disease ${ }^{22,24}$ or herald acute exacerbation. ${ }^{25}$ Various symptoms corresponding to a brainstem lesion may develop, such as nystagmus, dysarthria, dysphagia, ataxia, or ophthalmoplegia. ${ }^{15,20, \mathrm{e} 11, \mathrm{e} 12}$

Periependymal lesions surrounding the lateral ventricles. Lesions in the corpus callosum have been described in $12 \%$ to $40 \%$ of patients with NMOSD. ${ }^{12,15,26}$ Because both NMO and MS frequently have callosal lesions, location by itself is not a unique finding that differentiates NMOSD from MS. However, while the callosal lesions in MS are discrete, ovoid, and perpendicular to the ventricles and involve inferior aspects of the corpus callosum (figure 2A), ${ }^{\mathrm{e} 13, \mathrm{e} 14}$ NMOSD lesions are located immediately next to the lateral ventricles, following the ependymal lining (figure 1C.a). ${ }^{12}$ The acute callosal lesions in NMOSD are often edematous and heterogeneous, creating a "marbled pattern" 26 and sometimes involving the complete thickness of splenium in a unique "arch bridge pattern" (figure 1, C.b and C.c). ${ }^{12}$ Sometimes, the callosal lesions extend into the cerebral 

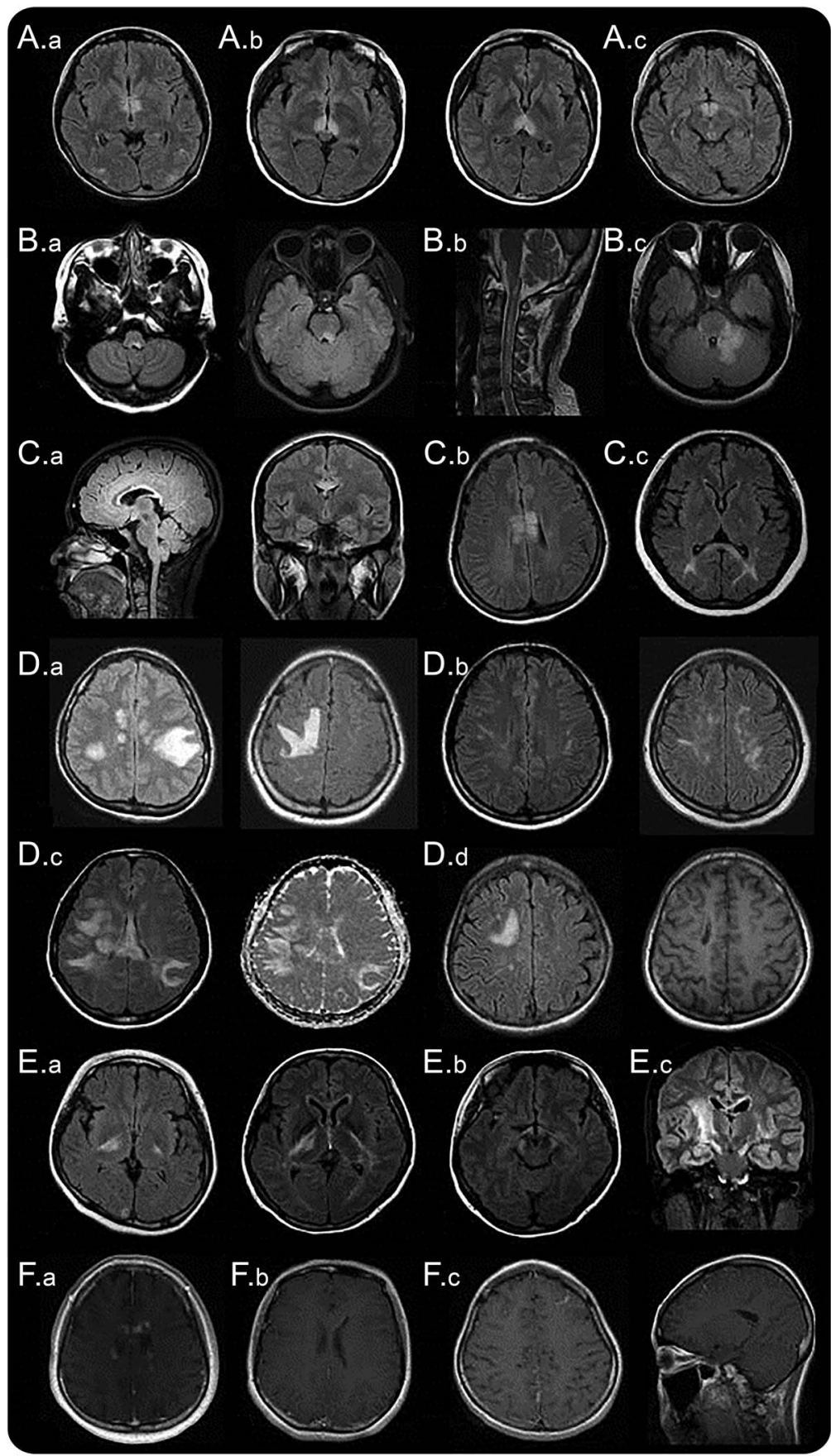

Diencephalic lesions surrounding (A.a) the third ventricles and cerebral aqueduct, (A.b) which include thalamus, hypothalamus, and (A.c) anterior border of the midbrain. (B.a) Dorsal brainstem lesion adjacent to the fourth ventricle, (B.b) linear medullary lesion that is contiguous with cervical cord lesion, (B.c) edematous and extensive dorsal brainstem lesion involving the cerebellar peduncle. (C.a) Callosal lesion immediately next to the lateral ventricle, following the ependymal lining, (C.b) "marbled pattern" callosal lesion, (C.c) "arch bridge pattern" callosal lesion. (D.a) Tumefactive hemispheric white matter lesions, (D.b) a long spindle-like or radial-shape lesion following white matter tracts, (D.c) extensive and confluent hemispheric lesions show increased diffusivity on apparent diffusion coefficient maps suggesting vasogenic edema, (D.d) hemispheric lesions in the chronic phase showing cystic-like cavitary changes. (E.a) Corticospinal tract lesions involving the posterior limb of the internal capsule and (E.b) cerebral peduncle of the midbrain, (E.c) longitudinally extensive lesion following the pyramidal tract. (F.a) Cloud-like enhancement, (F.b) linear enhancement of the ependymal surface of the lateral ventricles, (F.c) meningeal enhancement.

hemisphere, forming an extensive and confluent white matter lesion. ${ }^{12}$ In the chronic phase of NMOSD, the callosal lesions tend to reduce in size and intensity and may even disappear ${ }^{26}$; however, cystic changes and atrophy of the corpus callosum have been described. ${ }^{\text {e15 }}$ Certain clinical symptoms, such as dysfunctions of cognition and motor coordination, may be attributed to callosal lesions, but they have not been well evaluated yet.

Hemispheric white matter lesions. Extensive and confluent hemispheric white matter lesions are often tumefactive ( $>3 \mathrm{~cm}$ in longest diameter) or have long spindle-like or radial-shape following white matter tracts (figure 1D). ${ }^{12}$ Mass effect is usually absent. ${ }^{\text {e16 }}$ Increased lesion diffusivity on apparent diffusion coefficient maps suggests vasogenic edema in association with acute inflammation (figure 1D.c), ${ }^{12,27}$ occasionally mimicking posterior reversible encephalopathy syndrome ${ }^{28}$ or Baló lesions. ${ }^{\mathrm{e} 17, \mathrm{e} 18}$ These extensive lesions have been found more frequently in anti-AQP4 antibody seropositive than seronegative patients. ${ }^{29}$ In the chronic phase, these large lesions tend to shrink and even disappear, but in some cases, cystic-like or cavitary changes are revealed (figure 1D. d) ${ }^{\text {e19,e20 }}$ These lesions may cause various symptoms such as hemiparesis, encephalopathy, and visual field defects depending on the area they involve. Large confluent hemispheric white matter lesions are not uncommon in children with NMOSD. Tumefactive lesions with a surrounding zone of edema and variable mass effect may resemble acute disseminated encephalomyelitis $^{20,30}$ or CNS malignancies. ${ }^{31}$

Lesions involving corticospinal tracts. Lesions involving the corticospinal tracts can be unilateral or bilateral, and may extend from the deep white matter in the cerebral hemisphere through the posterior limb of the internal capsule to reach the cerebral peduncles of the midbrain or the pons (figure 1E). ${ }^{12}$ These lesions are contiguous and often longitudinally extensive, following the pyramidal tracts (figure 1E.c). Corticospinal tract lesions have been found in $23 \%$ to $44 \%$ in some cohorts of patients with $\mathrm{NMOSD}^{12, e 2}$ and have occasionally been reported in other cohorts. ${ }^{11,13}$ It is of interest that, unlike circumventricular areas, corticospinal tracts are not the areas where the AQP4 is highly expressed; it is unknown why these regions are also frequently involved in NMOSD.

Nonspecific lesions: Not unique, but most common. Nonspecific punctate or small $(<3 \mathrm{~mm})$ dots or patches of hyperintensities on T2-weighted or fluid-attenuated inversion recovery sequences in the subcortical or deep white matter have been described most frequently on brain imaging studies of NMOSD $(35 \%-84 \%)^{11,12,17}$ and are usually asymptomatic.

Enhancing lesions. Although the exact frequency is unclear, previous studies have described a variable 
Figure $2 \quad$ MRI lesions characteristic of MS
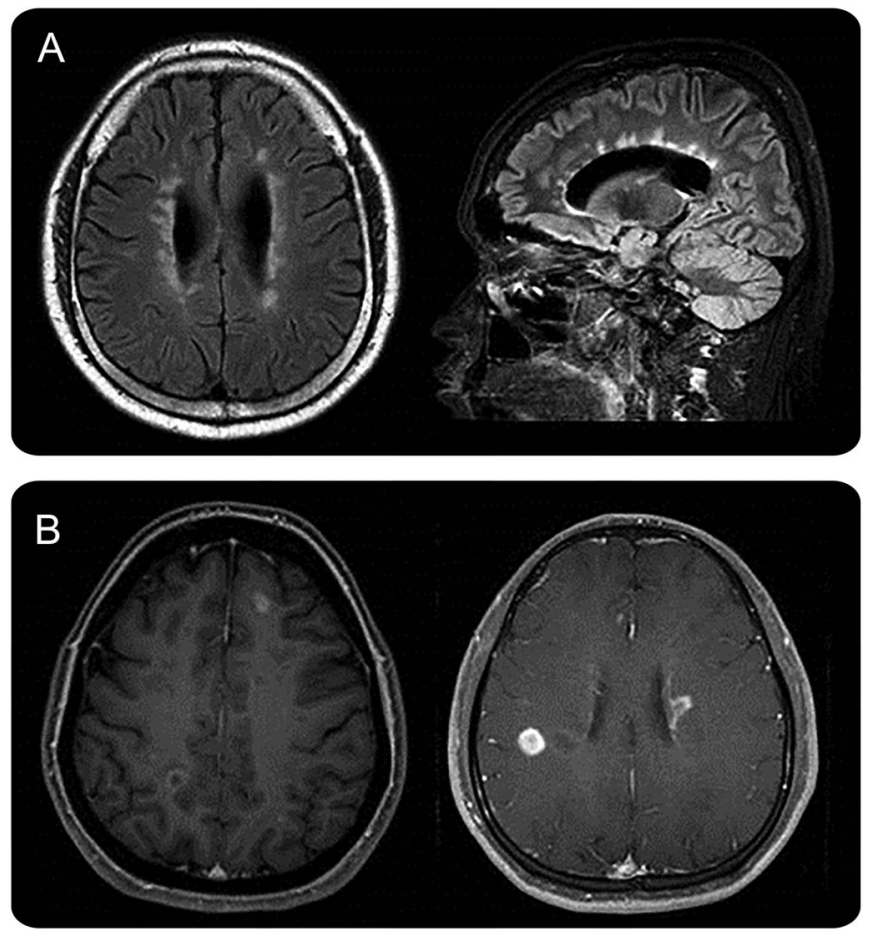

(A) Contrasting MS periventricular and callosal lesions, which are discrete, ovoid, and perpendicular to the ventricles. (B) Contrasting MS enhancing lesions, which are ovoid or open-ring gadolinium-enhanced lesions with well-defined borders. MS = multiple sclerosis.

percentage of gadolinium-enhancing brain lesions $(9 \%-36 \%)$ in patients with NMOSD. ${ }^{12,15, \mathrm{e} 2, \mathrm{e} 3}$ Most of the enhancement was displayed in a poorly marginated, subtle, and multiple patchy pattern, a socalled "cloud-like" enhancement (figure 1F.a). ${ }^{18}$ These cloud-like enhanced lesions differ from the ovoid or ring/open-ring gadolinium-enhancing lesions with well-defined borders that are more typical of MS (figure 2). A linear enhancement of the ependymal surface of the lateral ventricles (pencil-thin lesion) has also been described in NMOSD (figure 1F.b). ${ }^{21}$ Rarely, well-marginated nodular enhancement or meningeal enhancement has been reported in NMOSD (figure 1F.c). ${ }^{12, \mathrm{e} 16}$

OPTIC NERVE MRI FINDINGS IN NMOSD MRI studies have reported nonspecific optic nerve sheath thickening, optic nerve hyperintensities on T2weighted sequences, and gadolinium enhancement on T1-weighted sequences in acute $\mathrm{ON}$ of NMOSD. ${ }^{14,17}$ However, as similar findings also have been described in ON of MS, e22 these findings are not considered diagnostic of NMOSD. Recent studies have looked at the differential MRI features of the optic nerve lesion between MS and NMOSD. ${ }^{32,33}$ A trend to more posterior involvement of the optic nerve including chiasm, and simultaneous bilateral disease, has been observed in NMOSD (figure 3). ${ }^{32,33}$ Thus, long-segment inflammation of the optic nerve, particularly when simultaneous bilateral and extending posteriorly into the optic chiasm, should lead us to suspect the diagnosis of NMOSD in the appropriate clinical context.

SPINAL CORD MRI FINDINGS IN NMOSD The inflammatory process of NMOSD in spinal cord MRI is characterized by hyperintensity on T2weighted sequences and by hypointensity on T1weighted sequences. These abnormalities in the spinal cord MRI have been reported to be, in general, more frequently present in the cervical and the upper thoracic spinal cord segments than the lower thoracic and lumbar regions ${ }^{23,34, e 23}$ with a preferential involvement in the central gray matter. ${ }^{34,35}$ In the spinal cord, AQP4 is abundant in the gray matter and in glial cell processes adjacent to the ependymal cells of the central canal and to a lesser degree in the white matter of the spinal cord. ${ }^{24}$

The most distinct manifestation of NMO is LETM, defined as a lesion that spans over 3 or more contiguous vertebral segments and predominantly involves central gray matter on the spinal cord MRI (figure 4). ${ }^{4}$ However, not all LETM is NMOSD and several studies of patients with LETM have observed significant differences in demographic and clinical features between anti-AQP4 antibody positive compared with negative patients with LETM. ${ }^{19,36-38}$ LETM seems to be less specific for NMO in children than in adults. LETM is frequently observed in children with acute disseminated encephalomyelitis, ${ }^{39,40}$ but also in $17 \%$ of those with MS, ${ }^{\text {225 }}$ and in $67 \%$ to $88 \%$ of children with monophasic transverse myelitis. ${ }^{\text {e26,e27 }}$ Therefore, it is important to bear in mind that numerous other differential diagnoses than NMOSD need to be considered when a patient presents with LETM.

Spinal cord lesions during follow-up of NMOSD. MRI changes of LETM have been observed over the course of NMOSD and MRI data indicate that LETM lesions may evolve into multiple shorter lesions during remission or after treatment with high-dose steroids. ${ }^{23,41}$ In addition, spinal cord atrophy as a consequence of recurrent myelitis has been reported and may correlate with neurologic disability. ${ }^{23}$ Consequently, the timing of MRI may be important for the demonstration of LETM. ${ }^{42}$

COMPARING THE IMAGING OF NMOSD WITH MS In clinical practice, the main differential diagnosis of NMO is MS, particularly disease limited to the optic nerves and spinal cord. Differentiating these conditions is of prime importance because of differences in prognosis and therapy, as some MS therapies can exacerbate NMO. ${ }^{43-45}$ Thus, it is important to 
Figure 3 Optic nerve MRI lesions characteristic of neuromyelitis optica spectrum disorder
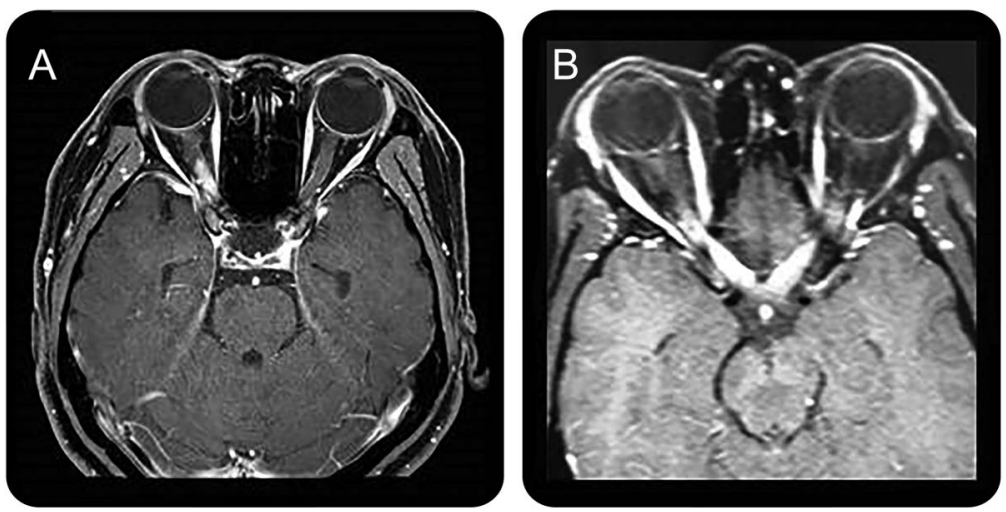

(A) Dense gadolinium-enhancing lesion at the posterior part of the right optic nerve. (B) Extensive gadolinium-enhancing lesion at the bilateral posterior part of the optic nerve/chiasm.

improve the methods and analysis by which to distinguish these conditions to facilitate early and accurate diagnosis. Contrasting features between the 2 conditions may further improve our understanding of the different pathogenic processes.

Whereas it is possible to select patients with NMOSD using the specific marker (serum antiAQP4 antibodies), there is no corresponding specific biomarker for MS. Studies contrasting NMO and MS have often used different selection criteria, particularly whether they have restricted the NMO inclusion criteria to patients positive for anti-AQP4 antibody or not, and this may influence the results. Conflicting data may also be partly explained by the use of various assays for anti-AQP4 antibodies, which

Figure 4 Spinal cord MRI lesions characteristic of neuromyelitis optica spectrum disorder

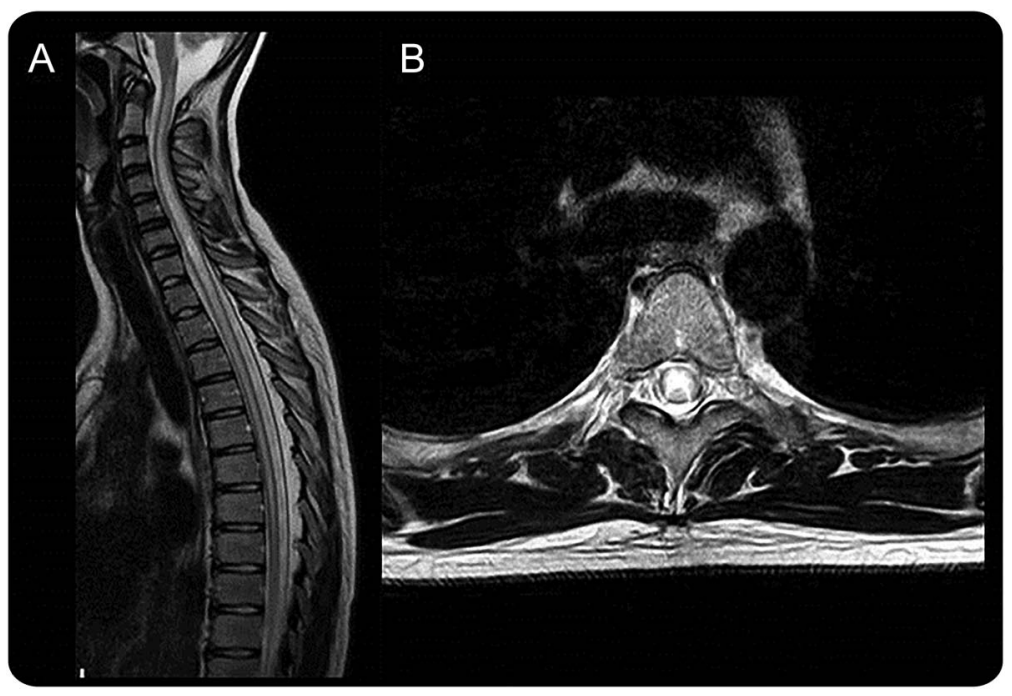

(A) Longitudinally extensive cord lesion involving thoracic cord. (B) Exclusive involvement of gray matter (H-shaped cord lesion). differ in sensitivity and are confounded by differences in the duration of follow-up.

As previously described, the most important imaging hallmark of NMO is the LETM, but a few patients may have centrally located short myelitis. ${ }^{46}$ Other MRI features of the spinal cord lesion that appear to differ between NMOSD and MS are summarized in the table.

The 2006 NMO diagnostic criteria include a brain MRI that is nondiagnostic for MS (using the Paty criteria) at onset as support for NMO. However, it is now known that MS-like lesions may appear in $10 \%$ to $12.5 \%$ of cases, ${ }^{11, e 3}$ and $5 \%$ to $42 \%$ of patients with NMO fulfill the Barkhof criteria. ${ }^{6,14,15,47} \mathrm{~A}$ recent report showed that $13 \%$ and $9 \%$ of patients with NMOSD, respectively, met Barkhof and the European Magnetic Imaging in MS diagnostic criteria for MS on brain MRI at onset. ${ }^{7}$ Lesion probability maps have not found statistically significant lesion locations in patients positive for anti-AQP4 antibody over those with MS. ${ }^{6}$ However, distinguishing features were identified on MS brain MRI that were sensitive and specific, such as the presence of a lateral ventricle and inferior temporal lobe lesion, Dawson fingers, or an S-shaped U-fiber lesion, to classify the patient as MS. Imaging sensitive to cortical lesions has revealed their absence in NMO (excluding one Japanese study of NMO pathology ${ }^{48}$ ), whereas they are seen in the majority of patients with MS.49,50 Characteristic MS brain lesions surround central venule in $>80 \%$ on high-strength MRI. ${ }^{50,51}$ In NMO lesions, this is less frequent, reported in $9 \%$ to $35 \%$ of cases $^{50,52}$ and likely indicates the different pathogenic mechanisms of the disease.

The frequency of silent lesion formation appears to differ between the 2 diseases. Patients with NMOSD are less likely to develop clinically silent MRI lesions than patients with MS. However, new silent MRI lesions do occur in a small proportion of patients with NMOSD. In addition, most studies show that nonlesional tissue damage as measured on nonconventional imaging such as diffusion tensor imaging is well recognized in MS and may not occur in $\mathrm{NMO}$ except in the connecting tracts up and downstream of lesions. ${ }^{53,54}$ Collectively, these findings support the clinical observation that NMO, in contrast to MS, may be a lesion-dependent disease that produces relapses without more generalized neurodegenerative pathology, and hence the lack of a progressive phase.

The differences noted between NMO and MS may relate to the CNS-specific antibody-mediated pathology against astrocytes rather than a T-cellpredominant inflammatory condition targeting myelin. In support of this possibility, a marker of astrocytic function, myo-inositol was reduced in 


\begin{tabular}{|lll|}
\hline Table & Comparison of characteristic MRI findings between NMOSD and MS & \\
\hline $\begin{array}{l}\text { Spinal } \\
\text { cord }\end{array}$ & NMOSD & MS \\
& Congitudinally extensive lesion ( $\geq 3$ vertebral segments) & Short, often multiple lesions \\
& T1 hypointensity common on acute lesions & Peripheral/asymmetrical/often posterior \\
$\begin{array}{l}\text { Optic } \\
\text { nerve }\end{array}$ & Long-length/posterior-chiasmal lesions & T1 hypointensity rare \\
Brain & $\begin{array}{l}\text { Periependymal lesions surrounding the ventricular system (wide-based } \\
\text { along the ependymal lining) }\end{array}$ & Short-length lesions \\
& Hemispheric tumefactive lesions & Dawson fingers (perpendicular to ventricles)/S-shaped U-fiber lesions, \\
& inferior lateral ventricle and temporal lobe lesions
\end{tabular}

Abbreviations: MRS = magnetic resonance spectroscopy; MS = multiple sclerosis; NMOSD = neuromyelitis optica spectrum disorder.

cervical cord lesions of patients positive for antiAQP4 antibody, but not in patients with MS. In contrast, $N$-acetyl-aspartate, a marker of myelinand neurofilament-specific injury, was significantly reduced in patients with MS compared with controls and nonsignificantly reduced in the patients positive for anti-AQP4 antibody. ${ }^{55}$

Important comparisons between NMOSD and MS scans are summarized in the table. Because long-term systematic imaging studies in NMO have not yet been performed, the reported cross-sectional differences compared with MS require further confirmation. Developing algorithms using the brain criteria described by Matthews et al. ${ }^{6}$ in combination with spinal cord and optic nerve imaging features and possibly nonconventional imaging may further improve the sensitivity and specificity.

PROGNOSTIC IMPLICATION OF MRI ABNORMALITIES Anti-AQP4 antibody positivity is established as a prognostic marker, and its positivity indicates a high risk of further relapses of $\mathrm{ON}$ and myelitis. ${ }^{56,57}$ Because of the presence of many imaging features suggesting severe damage of spinal cord, such as $\mathrm{T} 1$ hypointensity with edema or cavitation and atrophy, patients with NMOSD are more likely to have a poor recovery, refractory pain, ${ }^{\mathrm{e} 28}$ and a high risk of permanent disability. In addition, patients with NMOSD who have lesions in the upper cervical region extending to the brainstem may be at risk of respiratory failure.

High levels of glial fibrillary acidic protein in the CSF of patients with NMOSD during acute attacks correlated with length of MRI spinal cord lesion and Expanded Disability Status Scale score 6 months after those attacks. This correlation suggests that imaging findings may be proportional to the amount of astrocyte damage and have potential prognostic implications. ${ }^{229}$ The presence of extensive brain lesions might predict a higher rate of relapse and increased disability at follow-up. ${ }^{\text {30 }}$ Longitudinal follow-up studies are required to confirm whether patients with brain lesions have a worse prognosis than those without brain lesions. At this point, there are no individual MRI parameters that can predict the prognosis of NMO.

More recently, antibodies against myelinoligodendrocyte glycoprotein (MOG) have been found in some patients with clinical features of NMOSD, but who lack anti-AQP4 antibodies. ${ }^{58} \mathrm{~Pa}-$ tients exhibiting the anti-MOG-positive and antiAQP4-negative serotype have been suggested to have fewer attacks, bilateral ON, more caudal myelitis, and recover better than patients with anti-AQP4 antibodies and those who are seronegative for both antibodies. ${ }^{59,60}$ Therefore, patients presenting with an NMOSD phenotype with anti-MOG antibodies may have a distinct underlying disease mechanism presumably with a better prognosis than those with anti-AQP4 antibodies, although this needs to be confirmed by further studies.

OUTLOOK: MRI FINDINGS IN THE CONTEXT OF NMO DIAGNOSTIC CRITERIA The notion that brain MRI abnormalities are frequent in patients with NMOSD refutes the older doctrine that a normal brain MRI is a prerequisite for a diagnosis of NMO. Herein, we have reviewed the advances in our knowledge on the spectrum of imaging findings in NMOSD. However, sensitivity and specificity of these imaging features for NMOSD have not been systematically investigated in a prospective manner, 
and none of the findings can be considered pathognomonic or evidentiary for NMOSD. Therefore, as with other inflammatory CNS conditions, imaging findings should prompt broad differential diagnostic consideration - a topic that is beyond the scope of this review. The actual utility of lesion probability maps to distinguish NMO and MS is limited by an unclear definition of some traditional criteria for MSsuggestive findings, such as "Dawson fingers." 31 The picture is further complicated by recent observations of patients with anti-MOG antibodies that some, but not all, have considered part of the NMO spectrum. ${ }^{58,59}$ Some commonalities but also differences in clinical presentation, epidemiology, and imaging have been reported between these 2 conditions, suggesting that NMOSD may not be a homogeneous nosologic entity. In addition, because NMOSD can coexist with other autoimmune diseases and antibodies to other CNS antigens such as anti-NMDA receptor antibodies may be present in patients seropositive for anti-AQP4 antibody, it is possible that autoimmunity against multiple CNS autoantigens may participate in the formation of inflammatory lesions of NMOSD. ${ }^{\text {e32,es3 }}$ The emerging heterogeneity of NMOSD is mirrored by the broad range of neuroimaging findings summarized in this article. Areas for improved imaging that may facilitate more specific diagnostic, prognostic, therapeutic efficacy or other patient care benefit include higher-resolution imaging methods, 3-dimensional imaging of sitespecific lesions, and potential computationally guided analysis of images for quantitative comparisons. International collaborative efforts are now under way that will permit accrual of sufficiently large, carefully characterized NMO/NMOSD patients to better understand the frequency of brain involvement and to more thoroughly appreciate the implications of MRI abnormalities in clinical diagnosis and prognosis.

\section{AUTHOR CONTRIBUTIONS}

Dr. H.J. Kim conceived and designed the work, analyzed the literature, wrote the manuscript, critically reviewed and revised the manuscript, and approved the final manuscript. Dr. F. Paul conceived the work, analyzed the literature, wrote the manuscript, critically reviewed and revised the manuscript, and approved the final manuscript. Dr. M.A. Lana-Peixoto analyzed the literature, wrote the manuscript, critically reviewed and revised the manuscript, and approved the final manuscript. Dr. N. Asgari analyzed the literature, wrote the manuscript, critically reviewed and revised the manuscript, and approved the final manuscript. Dr. S. Tenembaum analyzed the literature, wrote the manuscript, critically reviewed and revised the manuscript, and approved the final manuscript. Dr. J. Palace analyzed the literature, wrote the manuscript, critically reviewed and revised the manuscript, and approved the final manuscript. Dr. E.C. Klawiter analyzed the literature, wrote the manuscript, critically reviewed and revised the manuscript, and approved the final manuscript. Dr. D.K. Sato analyzed the literature, wrote the manuscript, critically reviewed and revised the manuscript, and approved the final manuscript. Dr. J. de Seze critically reviewed and revised the manuscript, and approved the final manuscript. Dr. J. Wuerfel critically reviewed and revised the manuscript, and approved the final manuscript. Dr. B.L. Banwell critically reviewed and revised the manuscript, and approved the final manuscript. Dr. P. Villoslada critically reviewed and revised the manuscript, and approved the final manuscript. Dr. A. Saiz critically reviewed and revised the manuscript, and approved the final manuscript. Dr. K. Fujihara critically reviewed and revised the manuscript, and approved the final manuscript. Dr. S.-H. Kim analyzed the literature, wrote the manuscript, critically reviewed and revised the manuscript, and approved the final manuscript.

\section{ACKNOWLEDGMENT}

The authors thank The Guthy-Jackson Charitable Foundation for its support in organizing the NMO International Clinical Consortium \& Biorepository. The authors thank Drs. Brian Weinshenker and Jack Simon for their comments and Dr. Valerie Pasquetto for her assistance.

\section{STUDY FUNDING}

No targeted funding reported.

\section{DISCLOSURE}

H. Kim has given talks, consulted, and received honoraria and/or research support from Bayer Schering Pharma, Biogen Idec, Genzyme, Kael-GemVax, Merck Serono, Novartis, Teva-Handok, and UCB. He serves on a steering committee for MedImmune. F. Paul has received funding from German Research Council, German Ministry of Education and Research (Competence Network Multiple Sclerosis "KKNMS"), and The GuthyJackson Charitable Foundation. He has received travel compensation, speaker honoraria, and research support from Biogen, Bayer, Teva, Merck, Novartis, and Sanofi, and has served as steering committee member of the OCTIMS Study sponsored by Novartis. M. Lana-Peixoto reports no disclosures relevant to the manuscript. S. Tenembaum has provided consulting services to Genzyme Corporation and Biogen Idec and received lecture fees from Merck Serono. N. Asgari reports no disclosures relevant to the manuscript. J. Palace is partly funded by highly specialized services to run a national congenital myasthenia service and a neuromyelitis service. She has received support for scientific meetings and honorariums for advisory work from Merck Serono, Biogen Idec, Novartis, Teva, Chugai Pharma, and Bayer Schering, and unrestricted grants from Merck Serono, Novartis, Biogen Idec, and Bayer Schering. Her hospital trust receives funds for her role as clinical lead for the RSS, and she has received grants from the MS Society and Guthy-Jackson Foundation for unrelated research studies. E. Klawiter has received research funding from Roche. He has received consulting fees and/or speaking honoraria from Biogen Idec, Bayer Healthcare, Genzyme Corporation, and Teva Neuroscience. D. Sato receives scholarship funds from the Ministry of Education, Culture, Sports, Science and Technology (MEXT) of Japan and has received research support from Ichiro Kanehara Foundation. J. de Seze has received honoraria from Bayer Schering, Biogen Idec, LFB, Merck Serono, Novartis, Sanofi-Aventis, and Teva. He serves as a consultant for Alexion and Chugai. J. Wuerfel serves on advisory boards for Novartis and Biogen Idec. He received a research grant from Novartis, and speaker honoraria from Bayer, Novartis, and Biogen Idec. He is supported by the German Ministry of Science (BMBF/KKNMS). B. Banwell serves as a senior editor for Multiple Sclerosis and Related Disorders and on the editorial board of Neurology ${ }^{\circledR}$. She serves as a consultant for Biogen Idec, Novartis, Teva Neuroscience, and Merck Serono. She has been funded by the Canadian MS Research Foundation, the Canadian MS Society, and CIHR. P. Villoslada serves as a board member for Roche, Novartis, Neurotec Pharma, Bionure Farma, and as a consultant for Novartis, Roche, TFS, Heidelberg Engineering, MedImmune, Digna Biotech, and Neurotec Pharma. He has received research support from European Commission, Instituto Salud Carlos III, Marato TV3, Novartis, and Roche and travel expenses from Novartis. He holds patents with Digna Biotech, Bionure Farma, and stock/stock options of Bionure Farma. A. Saiz has received compensation for consulting services and speaking from Bayer Schering, Merck Serono, Biogen Idec, Sanofi-Aventis, Teva Pharmaceutical Industries, and Novartis. K. Fujihara serves on scientific advisory boards for Bayer Schering Pharma, Biogen Idec, Mitsubishi Tanabe Pharma Corporation, Novartis Pharma, Chugai Pharmaceutical, Ono Pharmaceutical, Nihon Pharmaceutical, Merck Serono, Alexion Pharmaceuticals, MedImmune, and Medical Review; has received funding for travel and speaker honoraria 
from Bayer Schering Pharma, Biogen Idec, Eisai Inc., Mitsubishi Tanabe Pharma Corporation, Novartis Pharma, Astellas Pharma Inc., Takeda Pharmaceutical Company Limited, Asahi Kasei Medical Co., Daiichi Sankyo, and Nihon Pharmaceutical; serves as an editorial board member of Clinical and Experimental Neuroimmunology (2009-present) and an advisory board member of Sri Lanka Journal of Neurology; has received research support from Bayer Schering Pharma, Biogen Idec Japan, Asahi Kasei Medical, The Chemo-Sero-Therapeutic Research Institute, Teva Pharmaceutical, Mitsubishi Tanabe Pharma, Teijin Pharma, Chugai Pharmaceutical, Ono Pharmaceutical, Nihon Pharmaceutical, and Genzyme Japan; and is funded as the secondary investigator (22229008, 2010-2015) by the Grants-in-Aid for Scientific Research from the Ministry of Education, Science and Technology of Japan and as the secondary investigator by the Grants-in-Aid for Scientific Research from the Ministry of Health, Welfare and Labor of Japan (2010-present). S. Kim reports no disclosures relevant to the manuscript. Go to Neurology.org for full disclosures.

Received June 29, 2014. Accepted in final form November 18, 2014.

\section{REFERENCES}

1. Wingerchuk DM, Hogancamp WF, O'Brien PC, Weinshenker BG. The clinical course of neuromyelitis optica (Devic's syndrome). Neurology 1999;53:1107-1114.

2. Lennon VA, Wingerchuk DM, Kryzer TJ, et al. A serum autoantibody marker of neuromyelitis optica: distinction from multiple sclerosis. Lancet 2004;364:2106-2112.

3. Wingerchuk DM, Lennon VA, Pittock SJ, Lucchinetti CF, Weinshenker BG. Revised diagnostic criteria for neuromyelitis optica. Neurology 2006;66:1485-1489.

4. Wingerchuk DM, Lennon VA, Lucchinetti CF, Pittock SJ, Weinshenker BG. The spectrum of neuromyelitis optica. Lancet Neurol 2007;6:805-815.

5. Kim SH, Kim W, Li XF, Jung IJ, Kim HJ. Clinical spectrum of CNS aquaporin-4 autoimmunity. Neurology 2012;78:1179-1185.

6. Matthews L, Marasco R, Jenkinson M, et al. Distinction of seropositive NMO spectrum disorder and MS brain lesion distribution. Neurology 2013;80:1330-1337.

7. Huh SY, Min JH, Kim W, et al. The usefulness of brain MRI at onset in the differentiation of multiple sclerosis and seropositive neuromyelitis optica spectrum disorders. Mult Scler 2014;20:695-704.

8. O'Riordan JI, Gallagher HL, Thompson AJ, et al. Clinical, CSF, and MRI findings in Devic's neuromyelitis optica. J Neurol Neurosurg Psychiatry 1996;60:382-387.

9. Filippi M, Rocca MA, Moiola L, et al. MRI and magnetization transfer imaging changes in the brain and cervical cord of patients with Devic's neuromyelitis optica. Neurology 1999;53:1705-1710.

10. Pittock SJ, Weinshenker BG, Lucchinetti CF, Wingerchuk DM, Corboy JR, Lennon VA. Neuromyelitis optica brain lesions localized at sites of high aquaporin 4 expression. Arch Neurol 2006;63:964-968.

11. Pittock SJ, Lennon VA, Krecke K, Wingerchuk DM, Lucchinetti CF, Weinshenker BG. Brain abnormalities in neuromyelitis optica. Arch Neurol 2006;63:390-396.

12. Kim W, Park MS, Lee SH, et al. Characteristic brain magnetic resonance imaging abnormalities in central nervous system aquaporin-4 autoimmunity. Mult Scler 2010; 16:1229-1236.

13. Bichuetti DB, Rivero RL, Oliveira DM, et al. Neuromyelitis optica: brain abnormalities in a Brazilian cohort. Arq Neuropsiquiatr 2008;66:1-4.

14. Wang F, Liu Y, Duan Y, Li K. Brain MRI abnormalities in neuromyelitis optica. Eur J Radiol 2011;80:445-449.
15. Chan KH, Tse CT, Chung CP, et al. Brain involvement in neuromyelitis optica spectrum disorders. Arch Neurol 2011;68:1432-1439.

16. Ghezzi A, Bergamaschi R, Martinelli V, et al. Clinical characteristics, course and prognosis of relapsing Devic's neuromyelitis optica. J Neurol 2004;251:47-52.

17. Cabrera-Gomez JA, Quevedo-Sotolongo L, GonzalezQuevedo A, et al. Brain magnetic resonance imaging findings in relapsing neuromyelitis optica. Mult Scler 2007;13: 186-192.

18. Ito S, Mori M, Makino T, Hayakawa S, Kuwabara S. "Cloud-like enhancement" is a magnetic resonance imaging abnormality specific to neuromyelitis optica. Ann Neurol 2009;66:425-428.

19. Jarius S, Ruprecht K, Wildemann B, et al. Contrasting disease patterns in seropositive and seronegative neuromyelitis optica: a multicentre study of 175 patients. J Neuroinflammation 2012;9:14.

20. McKeon A, Lennon VA, Lotze T, et al. CNS aquaporin-4 autoimmunity in children. Neurology 2008;71:93-100.

21. Popescu BF, Lennon VA, Parisi JE, et al. Neuromyelitis optica unique area postrema lesions: nausea, vomiting, and pathogenic implications. Neurology 2011;76:1229-1237.

22. Apiwattanakul M, Popescu BF, Matiello M, et al. Intractable vomiting as the initial presentation of neuromyelitis optica. Ann Neurol 2010;68:757-761.

23. Asgari N, Skejoe HP, Lillevang ST, Steenstrup T, Stenager E, Kyvik KO. Modifications of longitudinally extensive transverse myelitis and brainstem lesions in the course of neuromyelitis optica (NMO): a populationbased, descriptive study. BMC Neurol 2013;13:33.

24. Kim W, Kim SH, Lee SH, Li XF, Kim HJ. Brain abnormalities as an initial manifestation of neuromyelitis optica spectrum disorder. Mult Scler 2011;17:1107-1112.

25. Takahashi T, Miyazawa I, Misu T, et al. Intractable hiccup and nausea in neuromyelitis optica with antiaquaporin-4 antibody: a herald of acute exacerbations. J Neurol Neurosurg Psychiatry 2008;79:1075-1078.

26. Nakamura M, Misu T, Fujihara K, et al. Occurrence of acute large and edematous callosal lesions in neuromyelitis optica. Mult Scler 2009;15:695-700.

27. Matsushita T, Isobe N, Matsuoka T, et al. Extensive vasogenic edema of anti-aquaporin-4 antibody-related brain lesions. Mult Scler 2009;15:1113-1117.

28. Magana SM, Matiello M, Pittock SJ, et al. Posterior reversible encephalopathy syndrome in neuromyelitis optica spectrum disorders. Neurology 2009;72:712-717.

29. Matsushita T, Isobe N, Piao H, et al. Reappraisal of brain MRI features in patients with multiple sclerosis and neuromyelitis optica according to anti-aquaporin-4 antibody status. J Neurol Sci 2010;291:37-43.

30. Eichel R, Meiner Z, Abramsky O, Gotkine M. Acute disseminating encephalomyelitis in neuromyelitis optica: closing the floodgates. Arch Neurol 2008;65:267-271.

31. O’Mahony J, Bar-Or A, Arnold DL, et al. Masquerades of acquired demyelination in children: experiences of a national demyelinating disease program. J Child Neurol 2013;28:184-197.

32. Khanna S, Sharma A, Huecker J, Gordon M, Naismith RT, Van Stavern GP. Magnetic resonance imaging of optic neuritis in patients with neuromyelitis optica versus multiple sclerosis. J Neuroophthalmol 2012;32:216-220.

33. Storoni M, Davagnanam I, Radon M, Siddiqui A, Plant GT. Distinguishing optic neuritis in neuromyelitis 
optica spectrum disease from multiple sclerosis: a novel magnetic resonance imaging scoring system. J Neuroophthalmol 2013;33:123-127.

34. Cassinotto C, Deramond $\mathrm{H}$, Olindo S, Aveillan M, Smadja D, Cabre P. MRI of the spinal cord in neuromyelitis optica and recurrent longitudinal extensive myelitis. J Neuroradiol 2009;36:199-205.

35. Nakamura M, Miyazawa I, Fujihara K, et al. Preferential spinal central gray matter involvement in neuromyelitis optica: an MRI study. J Neurol 2008;255:163-170.

36. Kitley J, Leite MI, Kuker W, et al. Longitudinally extensive transverse myelitis with and without aquaporin 4 antibodies. JAMA Neurol 2013;70:1375-1381.

37. Chang KH, Lyu RK, Chen CM, et al. Distinct features between longitudinally extensive transverse myelitis presenting with and without anti-aquaporin 4 antibodies. Mult Scler 2013;19:299-307.

38. Sepulveda M, Blanco Y, Rovira A, et al. Analysis of prognostic factors associated with longitudinally extensive transverse myelitis. Mult Scler 2013;19:742-748.

39. Tenembaum S, Chitnis T, Ness J, Hahn JS; International Pediatric MSSG. Acute disseminated encephalomyelitis. Neurology 2007;68:S23-S36.

40. Banwell B, Tenembaum S, Lennon VA, et al. Neuromyelitis optica-IgG in childhood inflammatory demyelinating CNS disorders. Neurology 2008;70:344-352.

41. Krampla W, Aboul-Enein F, Jecel J, et al. Spinal cord lesions in patients with neuromyelitis optica: a retrospective long-term MRI follow-up study. Eur Radiol 2009;19: 2535-2543.

42. Iorio R, Damato V, Mirabella M, et al. Distinctive clinical and neuroimaging characteristics of longitudinally extensive transverse myelitis associated with aquaporin- 4 autoantibodies. J Neurol 2013;260:2396-2402.

43. Kim SH, Kim W, Li XF, Jung IJ, Kim HJ. Does interferon beta treatment exacerbate neuromyelitis optica spectrum disorder? Mult Scler 2012;18:1480-1483.

44. Min JH, Kim BJ, Lee KH. Development of extensive brain lesions following fingolimod (FTY720) treatment in a patient with neuromyelitis optica spectrum disorder. Mult Scler 2012;18:113-115.

45. Jacob A, Hutchinson M, Elsone L, et al. Does natalizumab therapy worsen neuromyelitis optica? Neurology 2012;79: 1065-1066.

46. Sato DK, Nakashima I, Takahashi T, et al. Aquaporin-4 antibody-positive cases beyond current diagnostic criteria for NMO spectrum disorders. Neurology 2013;80:2210-2216.
47. Asgari N, Lillevang ST, Skejoe HP, Falah M, Stenager E, Kyvik KO. A population-based study of neuromyelitis optica in Caucasians. Neurology 2011;76:1589-1595.

48. Saji E, Arakawa M, Yanagawa K, et al. Cognitive impairment and cortical degeneration in neuromyelitis optica. Ann Neurol 2013;73:65-76.

49. Calabrese M, Oh MS, Favaretto A, et al. No MRI evidence of cortical lesions in neuromyelitis optica. Neurology 2012;79:1671-1676.

50. Sinnecker T, Dorr J, Pfueller CF, et al. Distinct lesion morphology at 7-T MRI differentiates neuromyelitis optica from multiple sclerosis. Neurology 2012;79:708-714.

51. Kilsdonk ID, de Graaf WL, Soriano AL, et al. Multicontrast MR imaging at $7 \mathrm{~T}$ in multiple sclerosis: highest lesion detection in cortical gray matter with 3D-FLAIR. AJNR Am J Neuroradiol 2013;34:791-796.

52. Kister I, Herbert J, Zhou Y, Ge Y. Ultrahigh-field MR (7 $\mathrm{T})$ imaging of brain lesions in neuromyelitis optica. Mult Scler Int 2013;2013:398259.

53. Pichiecchio A, Tavazzi E, Poloni G, et al. Advanced magnetic resonance imaging of neuromyelitis optica: a multiparametric approach. Mult Scler 2012;18:817-824.

54. Klawiter EC, Xu J, Naismith RT, et al. Increased radial diffusivity in spinal cord lesions in neuromyelitis optica compared with multiple sclerosis. Mult Scler 2012;18: 1259-1268.

55. Ciccarelli O, Thomas DL, De Vita E, et al. Low myoinositol indicating astrocytic damage in a case series of neuromyelitis optica. Ann Neurol 2013;74:301-305.

56. Matiello M, Lennon VA, Jacob A, et al. NMO-IgG predicts the outcome of recurrent optic neuritis. Neurology 2008;70:2197-2200.

57. Weinshenker BG, Wingerchuk DM, Vukusic S, et al. Neuromyelitis optica IgG predicts relapse after longitudinally extensive transverse myelitis. Ann Neurol 2006;59: 566-569.

58. Kitley J, Woodhall M, Waters P, et al. Myelin-oligodendrocyte glycoprotein antibodies in adults with a neuromyelitis optica phenotype. Neurology 2012;79:1273-1277.

59. Sato DK, Callegaro D, Lana-Peixoto MA, et al. Distinction between MOG antibody-positive and AQP4 antibody-positive NMO spectrum disorders. Neurology 2014;82:474-481.

60. Kitley J, Waters P, Woodhall M, et al. Neuromyelitis optica spectrum disorders with aquaporin-4 and myelinoligodendrocyte glycoprotein antibodies: a comparative study. JAMA Neurol 2014;71:276-283.

\section{Subspecialty Alerts by E-mail!}

Customize your online journal experience by signing up for e-mail alerts related to your subspecialty or area of interest. Access this free service by visiting Neurology.org/site/subscriptions/etoc.xhtml or click on the "E-mail Alerts" link on the home page. An extensive list of subspecialties, methods, and study design choices will be available for you to choose from-allowing you priority alerts to cutting-edge research in your field! 


\section{Neurology}

\section{MRI characteristics of neuromyelitis optica spectrum disorder: An international update}

Ho Jin Kim, Friedemann Paul, Marco A. Lana-Peixoto, et al.

Neurology 2015;84;1165-1173 Published Online before print February 18, 2015

DOI 10.1212/WNL.0000000000001367

This information is current as of February 18, 2015

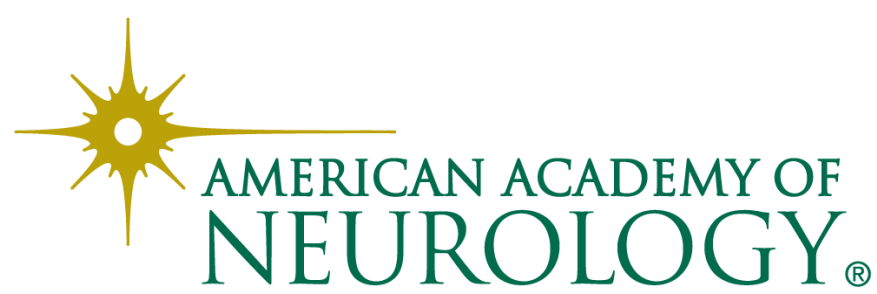




\section{Updated Information \& Services}

\section{Supplementary Material}

\section{References}

Citations

Subspecialty Collections

Permissions \& Licensing

\section{Reprints}

including high resolution figures, can be found at: http://n.neurology.org/content/84/11/1165.full

Supplementary material can be found at: http://n.neurology.org/content/suppl/2015/02/18/WNL.0000000000001 367.DC1

http://n.neurology.org/content/suppl/2015/02/18/WNL.0000000000001 367.DC2

This article cites 60 articles, 21 of which you can access for free at: http://n.neurology.org/content/84/11/1165.full\#ref-list-1

This article has been cited by 17 HighWire-hosted articles: http://n.neurology.org/content/84/11/1165.full\#\#otherarticles

This article, along with others on similar topics, appears in the following collection(s):

Devic's syndrome

http://n.neurology.org/cgi/collection/devics_syndrome

MRI

http://n.neurology.org/cgi/collection/mri

Multiple sclerosis

http://n.neurology.org/cgi/collection/multiple_sclerosis

Optic neuritis; see Neuro-ophthalmology/Optic Nerve

http://n.neurology.org/cgi/collection/optic_neuritis

Transverse myelitis

http://n.neurology.org/cgi/collection/transverse_myelitis

Information about reproducing this article in parts (figures,tables) or in its entirety can be found online at:

http://www.neurology.org/about/about_the_journal\#permissions

Information about ordering reprints can be found online:

http://n.neurology.org/subscribers/advertise

Neurology ${ }^{\circledR}$ is the official journal of the American Academy of Neurology. Published continuously since 1951, it is now a weekly with 48 issues per year. Copyright @ 2015 American Academy of Neurology. All rights reserved. Print ISSN: 0028-3878. Online ISSN: 1526-632X.

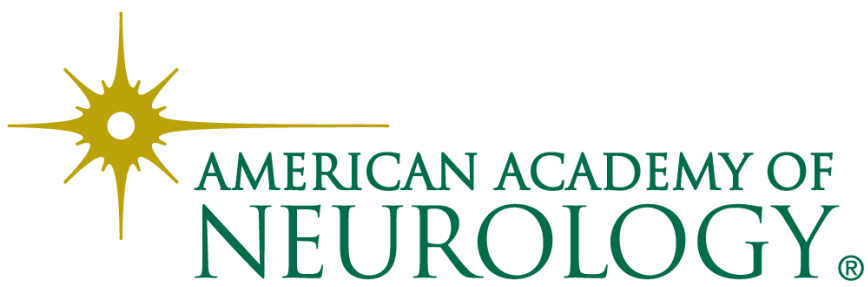

